\title{
Conservation of canonical circulation and its relation to finite Hall term magnetohydrodynamics
}

\author{
P. M. Bellan \\ California Institute of Technology, Pasadena, California 91125
}

(Received 25 January 1993; accepted 22 March 1993)

\begin{abstract}
The axisymmetric, compressible visco-resistive two-fluid plasma equations are examined under the constraint that the current is purely poloidal and the pressure is a function of density only ("barotropic"). For ideal plasmas (zero resistivity and zero viscosity) the Kelvin circulation theorem of fluid mechanics and the concept of frozen-in field lines turn out to be limiting cases of a more general concept, namely, that the canonical circulation $S_{\sigma}=\oint\left(m_{\sigma} \mathbf{u}_{\sigma}+q_{\sigma} \mathbf{A}\right) \cdot d \mathbf{l}$ of a toroidal fluid element, is exactly conserved as the toroidal element convects and/or is compressed. Appropriate linear combinations of the electron and ion fluid equations give a magnetohydrodynamic vorticity transport equation and an induction equation with a nonlinear Hall term. The finite Hall term is identical to the source term in the vorticity transport equation [P. M. Bellan, Phys. Rev. Lett. 69, 3515 (1992)], except for a constant factor related to the ion collisionless skin depth.
\end{abstract}

\section{INTRODUCTION}

An important class of plasmas are those which are azimuthally symmetric and where the magnetic field is purely toroidal, i.e., the current density is purely poloidal and the magnetic helicity is zero. Examples include $Z$ pinches, ${ }^{1-3}$ Marshall guns, ${ }^{4}$ dense plasma focus, ${ }^{5,6}$ arcs, ${ }^{7-10}$ magnetoplasmadynamic thrusters, ${ }^{11,12}$ ignitrons, thyratrons ${ }^{13}$ lightning, plasma erosion opening switches (PEOS), ${ }^{14,15}$ and possibly the "central engine" portion of astrophysical jets. ${ }^{16,17}$ Excluded are axisymmetric plasmas with purely poloidal currents such as $\theta$-pinches, and axisymmetric plasmas with finite magnetic helicity such as reversed-field pinches, tokamaks, and spheromaks. Also excluded are nonaxisymmetric plasmas such as stellarators.

Kulsrud et al. ${ }^{18}$ and Fruchtman ${ }^{19}$ have analyzed the generalized Ohm's law under the assumption that only the electron fluid convects ${ }^{20}$ and showed that in a nondissipative plasma the quantity $B / n r$ is convected with the electron fluid velocity $\mathbf{u}_{e}$. On the other hand, the author recently showed ${ }^{21}$ that for the magnetohydrodynamic (MHD) model where Hall terms are dropped, but ion fluid motion is included, in a nondissipative plasma the quantities $I / r^{2}$ and $\chi / r^{2}$ are both convected with the center of mass velocity $\mathbf{U}$, and furthermore, that axial gradients in current, $\partial I^{2} / \partial z$, act as volumetric sources for the vorticity related quantity $\chi$.

The purpose of this paper is to extend Ref. 21 to include both Hall terms and compressibility and to show the relationship of the resulting model to the concepts in Refs. 19 and 20. Two main conclusions result from this analysis:

(1) The Kelvin circulation theorem of fluid mechanics becomes generalized in two-fluid theory to a form where a new quantity, the canonical circulation, is convected with each fluid species.

(2) The Hall MHD equations have a nearly symmetric form where the nonlinear Hall term in the induction equation is the same as the vorticity source term in the vorticity transport equation (except for a constant factor related to the ion collisionless skin depth). Thus, the source of Hallrelated effects is essentially identical (except for the constant factor) to the source of vorticity.

The analysis here will consider both two-fluid and MHD models since both points of view provide useful insights. Plasma compressibility will be allowed with the proviso that the pressure is a specified function of density; this simplification is called the barotropic assumption, and allows one to avoid using a heat equation. The paper is organized as follows: Sec. II discusses the standard mathematical form of the equation of motion in this geometry and shows the standard manipulations that may be made. In Sec. III the basic two-fluid quantities are presented and related to each other. The general visco-resistive equations of motion for each species are discussed and equations involving vorticity and compressibility are developed. These two-fluid equations provide some interesting insights into the behavior of the plasma, but in general are too complicated to solve directly. In Sec. IV the two-fluid equations are transformed into compressible MHD equations with Hall terms; these are much more amenable to analysis than the two-fluid equations and are compared to the incompressible result of Ref. 21 and also to electron hydrodynamics. In Sec. V a summary is presented.

\section{GENERAL FORM OF EQUATIONS AND OF OPERATIONS}

In general, any fluid equation of motion will contain at least one term that is a gradient of a scalar potential (e.g., a pressure gradient or an electrostatic electric field). Because of axisymmetry this term has no $\theta$ component, and so the general form of the equation of motion must be

$$
\nabla g+\mathbf{Q} \times \nabla \theta=0,
$$

where $g$ is the sum of all the scalar potentials, and $\mathbf{Q}$ is 


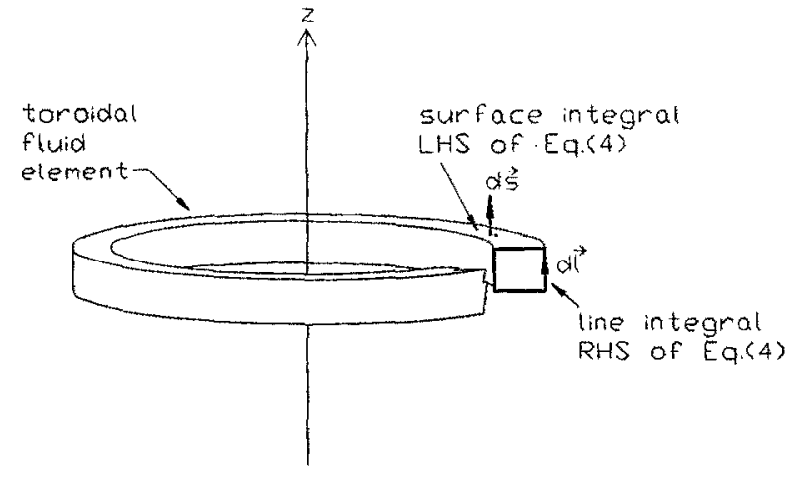

FIG. 1. Toroidal volume element used for Eq. (4); the surface integral is over the surface of the toroidal volume element (see ds vector in the figure), the line integral is around the poloidal circumference of the toroidal volume element (see dl vector in the figure).

some vector such that $\mathbf{Q} \times \nabla \theta$ is the sum of all the other terms in the equation of motion. Two general types of operation are possible on $\mathrm{Eq}$. (1):

(1) By first taking the curl of Eq. (1) and then dotting with $\nabla \theta$ gives

$$
\boldsymbol{\nabla} \cdot\left(\frac{1}{r^{2}} \mathbf{Q}\right)=0
$$

which is in a form convenient for flux conserving numerical integration schemes.

(2) The divergence of Eq. (1) gives

$$
\nabla^{2} g=-\nabla \theta \cdot \nabla \times \mathbf{Q}
$$

An alternate form, particularly suitable for flux conserving numerical integration schemes, is obtained by integrating Eq. (3) over the volume of a toroidal fluid element as shown in Fig. 1, giving

$$
\int d \mathbf{s} \cdot \nabla g=-2 \pi \oint d \mathbf{l} \cdot \mathbf{Q}
$$

where the surface integral on the left-hand side is over the surface of the toroidal element, and the line integral on the right-hand side is around the minor circumference.

Hence, no matter how complicated the equation of motion is, it will always be possible to identify $g$ and $Q$ and to obtain equations of the form of Eq. (2) and Eqs. (3) or (4). Taking the curl gives relationships between the rotational quantities (current, vorticity) while taking the divergence gives relationships between the irrotational quantities (pressure, electrostatic potential). For incompressible situations the rotational quantities are independent of the irrotational quantities and may be calculated without reference to the irrotational quantities, but in the general compressible situation there is significant coupling and irrotational and rotational quantities have to be calculated simultaneously.

\section{TWO-FLUID ANALYSIS}

\section{A. Standard form of compressible equations of motion}

In two-fluid theory the equation of motion for each plasma species $\sigma$ is

$$
\begin{aligned}
m_{\sigma}\left(\frac{\partial \mathbf{u}_{\sigma}}{\partial t}+\mathbf{u}_{\sigma} \cdot \nabla \mathbf{u}_{\sigma}\right)= & q_{\sigma}\left[\mathbf{E}+\mathbf{u}_{\sigma} \times \mathbf{B}\right]-\frac{1}{n_{\sigma}} \nabla P_{\sigma} \\
& -q_{\sigma} \eta \mathbf{J}-m_{\sigma} \nu_{\sigma} \nabla \cdot \boldsymbol{\Lambda}
\end{aligned}
$$

where $\eta$ is the plasma electrical resistivity, $v_{\sigma}$ is the kinematic viscosity, and in Cartesian coordinates

$$
\Lambda_{i j}=-\left(\frac{\partial u_{i}}{\partial x_{j}}+\frac{\partial u_{j}}{\partial x_{i}}-\frac{2}{3} \delta_{i j} \frac{\partial u_{j}}{\partial x_{i}}\right)
$$

so that

$$
\nabla \cdot \boldsymbol{A}=\nabla \times\left(\nabla \times \mathbf{u}_{\sigma}\right)-\frac{4}{3} \nabla\left(\nabla \cdot \mathbf{u}_{\sigma}\right) .
$$

For simplicity, we will ignore spatial dependence of viscosity in this analysis, but will retain spatial dependence for resistivity. The continuity equation for each species is

$$
\frac{\partial n_{\sigma}}{\partial t}+\mathbf{u}_{\sigma} \cdot \nabla n_{\sigma}+n_{\sigma} \nabla \cdot \mathbf{u}_{\sigma}=0
$$

We now assume that the temperature is a function of density, so that the pressure, which in general is a function of both deasity and temperature, becomes a function of density only, ${ }^{20}$ i.e., $P_{\sigma}=P_{\sigma}\left(n_{\sigma}\right)$. This is certainly true within a fluid element if the plasma is either adiabatic or isothermal, however, our assumption is more restrictive, because we are requiring that the same functional dependence hold for different fluid elements. With this assumption the pressure term in the equation of motion can be written

$$
\frac{1}{n_{\sigma}} \nabla P_{\sigma}\left(n_{\sigma}\right)=\nabla \Theta_{\sigma}\left(n_{\sigma}\right),
$$

where $\Theta_{\sigma}$ is a temperature-like scalar. The equation of motion can be recast as

$$
\begin{aligned}
m_{\sigma}( & \left.\frac{\partial \mathbf{u}_{\sigma}}{\partial t}+\nabla \frac{u_{\sigma}^{2}}{2}-\mathbf{u}_{\sigma} \times \nabla \times \mathbf{u}_{\sigma}\right) \\
= & q_{\sigma}\left(\mathbf{E}+\mathbf{u}_{\sigma} \times \mathbf{B}\right)-\nabla \Theta_{\sigma}-q_{\sigma} \eta \mathbf{J} \\
& \quad-m_{\sigma} v_{\sigma}\left[\nabla \times\left(\nabla \times \mathbf{u}_{\sigma}\right)-\frac{4}{3} \nabla\left(\nabla \cdot \mathbf{u}_{\sigma}\right)\right] .
\end{aligned}
$$

At this point it is useful to express the velocity in terms of a streamfunction $\psi_{\sigma}$ and a compressibility "potential" $\Omega_{\sigma}$, i.e.,

$$
\mathbf{u}_{\sigma}=\frac{1}{2 \pi}\left(\nabla \psi_{\sigma} \times \nabla \theta\right)-\nabla \Omega_{\sigma}
$$

and to define the "vorticity" scalar $\chi_{\sigma}$ by

$$
\nabla \times \mathbf{u}_{\sigma}=\frac{1}{2 \pi} \chi_{\sigma} \nabla \theta
$$

and a compressibility "source" $\Gamma_{\sigma}$ by

$$
\nabla \cdot \mathbf{u}_{\sigma}=\Gamma_{\sigma}
$$


(to provide more symmetric equations, the definition of $\chi_{\sigma}$ here differs from that in Ref. 21 by a factor of $2 \pi$ ). Explicitly substituting Eq. (11) into Eq. (12) gives a Poisson-like equation showing $\chi_{\sigma}$ as a source for $\psi_{\sigma}$

$$
r^{2} \nabla \cdot\left(\frac{1}{r^{2}} \nabla \psi_{\sigma}\right)=-\chi_{\sigma}
$$

and similar substitution into Eq. (13) gives the Poissonlike equation showing $\Gamma_{\sigma}$ as a source for $\Omega_{\sigma}$

$$
\nabla^{2} \Omega_{\sigma}=-\Gamma_{\sigma} .
$$

We assume that the plasma is neutral so that $Z n_{i}$ $=n_{e}$ and also $\nabla \cdot \mathbf{J}=0$. The current and velocities are related to each other by

$$
\mathbf{J}=n_{e} e\left(\mathbf{u}_{i}-\mathbf{u}_{e}\right)
$$

so that plasma neutrality provides the constraint that

$$
\left(\mathbf{u}_{i}-\mathbf{u}_{e}\right) \cdot \nabla n_{e}+n_{e}\left(\Gamma_{i}-\Gamma_{e}\right)=0 .
$$

The magnetic field and current density are, respectively,

$$
\mathbf{B}=\nabla \times \mathbf{A}=\frac{\mu_{0} I}{2 \pi} \nabla \theta
$$

and

$$
\mathbf{J}=\frac{1}{2 \pi} \nabla \mathbf{I} \times \nabla \theta .
$$

Note the isomorphism between the vector potential $\mathbf{A}$ and the fluid velocity $\mathbf{u}_{\sigma}$, between the vorticity $\chi$ and the current $\mu_{0} I$, and between the current density and the velocity. Furthermore, we choose Coulomb gauge so that

$$
\mathbf{A}=\frac{1}{2 \pi} \nabla f \times \nabla \theta
$$

in which case the electric field can be expressed as

$$
\mathbf{E}=-\nabla \phi-\left(\frac{1}{2 \pi} \nabla \frac{\partial f}{\partial t} \times \nabla \theta\right),
$$

where $\phi$ is the electrostatic potential. Explicit substitution of Eq. (20) into Eq. (18) gives the relation

$$
r^{2} \nabla \cdot\left(\frac{1}{r^{2}} \nabla f\right)=-\mu_{0} I
$$

which is isomorphic to Eq. (14).With these definitions, the equation of motion for each species can be written as

$$
\begin{aligned}
& \nabla\left[m_{\sigma}\left(-\frac{\partial \Omega_{\sigma}}{\partial t}+\frac{u_{\sigma}^{2}}{2}-\frac{4}{3} v_{\sigma} \nabla \cdot \mathbf{u}_{\sigma}\right)+q_{\sigma} \phi+\Theta_{\sigma}\right] \\
& +\frac{1}{2 \pi}\left[\begin{array}{c}
m_{\sigma}\left(\nabla \frac{\partial \psi_{\sigma}}{\partial t}-\chi_{\sigma} \mathbf{u}_{\sigma}+v_{\sigma} \nabla \chi_{\sigma}\right) \\
+q_{\sigma}\left(\nabla \frac{\partial f}{\partial t}-\mu_{0} I \mathbf{u}_{\sigma}+\eta \nabla I\right)
\end{array}\right] \times \nabla \theta=0 .
\end{aligned}
$$

As predicted this is in the form of Eq. (1).

\section{B. Rotational two-fluid equations}

As discussed in Sec. II, operating on Eq. (23) with $\nabla \theta \cdot \nabla \times$ gives an equation of the form $\nabla \cdot\left(r^{-2} \mathbf{Q}\right)=0$, namely

$$
\begin{aligned}
& m_{\sigma}\left[-\frac{\partial}{\partial t} \nabla \cdot\left(\frac{1}{r^{2}} \nabla \psi_{\sigma}\right)+\nabla \cdot\left(\frac{\chi_{\sigma}}{r^{2}} \mathbf{u}_{\sigma}\right)-\nabla \cdot\left(\frac{\nu_{\sigma}}{r^{2}} \nabla \chi_{\sigma}\right)\right] \\
& \quad+q_{\sigma}\left[-\frac{\partial}{\partial t} \boldsymbol{\nabla} \cdot\left(\frac{1}{r^{2}} \nabla f\right)+\nabla \cdot\left(\frac{\mu_{0} I}{r^{2}} \mathbf{u}_{\sigma}\right)-\nabla \cdot\left(\frac{\eta}{r^{2}} \nabla I\right)\right]=0 .
\end{aligned}
$$

Using Eqs. (14) and (22) and expanding the middle terms this becomes

$$
\begin{aligned}
& m_{\sigma}\left[\frac{\partial}{\partial t}\left(\frac{\chi_{\sigma}}{r^{2}}\right)+\mathbf{u}_{\sigma} \cdot \nabla\left(\frac{\chi_{\sigma}}{r^{2}}\right)+\frac{\chi_{\sigma}}{r^{2}} \nabla \cdot \mathbf{u}_{\sigma}-\nabla \cdot\left(\frac{\nu_{\sigma}}{r^{2}} \nabla \chi\right)\right] \\
& \quad+q_{\sigma}\left[\frac{\partial}{\partial t}\left(\frac{\mu_{0} I}{r^{2}}\right)+\mathbf{u}_{\sigma} \cdot \nabla\left(\frac{\mu_{0} I}{r^{2}}\right)+\frac{\mu_{0} I}{r^{2}} \nabla \cdot \mathbf{u}_{\sigma}-\nabla \cdot\left(\frac{\eta}{r^{2}} \nabla I\right)\right] \\
& \quad=0 .
\end{aligned}
$$

The first three vorticity convective terms can be rearranged using the continuity equation to eliminate $\boldsymbol{\nabla} \cdot \mathbf{u}_{\sigma}$ as follows

$$
\begin{aligned}
& \frac{\partial}{\partial t}\left(\frac{\chi_{\sigma}}{r^{2}}\right)+\mathbf{u}_{\sigma} \cdot \nabla\left(\frac{\chi_{\sigma}}{r^{2}}\right)+\frac{\chi_{\sigma}}{r^{2}} \nabla \cdot \mathbf{u}_{\sigma} \\
& \quad=\frac{\partial}{\partial t}\left(\frac{\chi_{\sigma}}{r^{2}}\right)+\mathbf{u}_{\sigma} \cdot \nabla\left(\frac{\chi_{\sigma}}{r^{2}}\right)-\frac{\chi_{\sigma}}{n_{\sigma} r^{2}}\left(\frac{\partial n_{\sigma}}{\partial t}+\mathbf{u}_{\sigma} \cdot \nabla n_{\sigma}\right) \\
& \quad=n_{\sigma}\left[\frac{\partial}{\partial t}\left(\frac{\chi_{\sigma}}{n_{\sigma} r^{2}}\right)+\mathbf{u}_{\sigma} \cdot \nabla\left(\frac{\chi_{\sigma}}{n_{\sigma} r^{2}}\right)\right] .
\end{aligned}
$$

Making a similar rearrangement of the first three current terms give

$n_{\sigma} \frac{d}{d t}\left(\frac{m_{\sigma} \chi_{\sigma}+q_{\sigma} \mu_{0} I}{n_{\sigma} r^{2}}\right)=m_{\sigma} \nabla \cdot\left(\frac{v_{\sigma}}{r^{2}} \nabla \chi\right)+q_{\sigma} \nabla \cdot\left(\frac{\eta}{r^{2}} \nabla I\right)$

where $d / d t=\partial / \partial t+\mathbf{u}_{\sigma} \cdot \nabla$ is the convective derivative for species $\sigma$. Thus, if the plasma is nondissipative (i.e., $v_{\sigma}$ $=0$ and $\eta=0)$ the quantity $\left(m_{\sigma} \chi_{\sigma}+q_{\sigma} \mu_{0} I\right) / n_{\sigma} r^{2}$ is convected with the respective fluid motion of each species. If we consider a thin toroidal fluid element of species $\sigma$ with major radius $r$ and minor cross section $a$, the number of particles in this element is $N=2 \pi r a n_{\sigma}$. As the toroidal element convects in the $r-z$ plane, $N$ remains constant so that

$$
a=\frac{N}{2 \pi r n_{\sigma}} .
$$

The fluid circulation $C$ around the minor cross section is

$$
C_{\sigma}=\int d \mathbf{s} \cdot \nabla \times \mathbf{u}_{\sigma}=\frac{a \chi_{\sigma}}{2 \pi r}=\frac{N \chi_{\sigma}}{(2 \pi)^{2} n_{\sigma^{2}}}
$$

while the toroidal flux through the cross section is

$$
\Phi=\int d \mathbf{s} \cdot \mathbf{B}=\frac{a \mu_{0} I}{2 \pi r}=\frac{N \mu_{0} I}{(2 \pi)^{2} n_{o} r^{2}} .
$$


Thus, in a nondissipative (i.e., ideal) plasma, Eq. (27) becomes

$$
\frac{d}{d t}\left(m_{\sigma} C_{\sigma}+q_{\sigma} \Phi\right)=0 .
$$

In ordinary hydrodynamics the Kelvin circulation theorem states that $d C / d t=0$ so that Eq. (31) is the electromagnetic generalization of the Kelvin circulation theorem. The quantity $m_{\sigma} C_{\sigma}+q_{\sigma} \Phi$ may justifiably be called the canonical circulation, in analogy to the way that ordinary mechanical momentum $m v$ of a particle becomes generalized to canonical momentum $m v+q \mathrm{~A}$ in the presence of a magnetic field.

The meaning of the canonical circulation can be examined further by using Stoke's theorem to rewrite Eqs. (29) and (30) as

$$
C_{\sigma}=\oint \mathbf{u}_{\sigma} \cdot d \mathbf{l}, \quad \Phi=\oint \mathbf{A} \cdot d \mathbf{l}
$$

so that Eq. (31) may be written as

$$
\frac{d S_{\sigma}}{d t}=0
$$

where

$$
S_{\sigma}=\oint \mathbf{p}_{\sigma} \cdot d \mathbf{l}
$$

is the action integral associated with the fluid element and $\mathbf{p}_{\sigma}=m_{\sigma} \mathbf{u}_{\sigma}+q_{\sigma} \mathbf{A}$ is the canonical momentum of the fluid flow in the $r-z$ plane. The fact that this action integral is preserved appears to be related to the concept of adiabatic invariance for single particle motion, since for periodic single particle motion, the action integral is an invariant of the motion, providing the system changes on a time scale slow compared to the period. Surprisingly, the concept of slowness has not been explicitly used in the derivation of Eq. (33) which thus appears to be true for arbitrarily fast time scales.

[Note: Hassam and $\mathrm{Huba}^{22}$ derived the ion form of Eq. (33) using a large Larmor radius assumption and, in a stationary plasma with straight magnetic field geometry, explored the implications for both normal modes and stability.]

\section{Irrotational two-fluid equations}

We next take the divergence of Eq. (23) to obtain the time evolution of the compressibility scalar

$$
\begin{aligned}
& m_{\sigma}\left(\frac{\partial}{\partial t} \Gamma_{\sigma}+\mathbf{u}_{\sigma} \cdot \nabla \Gamma_{\sigma}+\nabla^{2} \frac{u_{\sigma}^{2}}{2}-\mathbf{u}_{\sigma} \cdot \nabla^{2} \mathbf{u}_{\sigma}\right. \\
& \left.\quad-\left|\nabla \times \mathbf{u}_{\sigma}\right|^{2}-\frac{4 v_{\sigma}}{3} \nabla^{2} \Gamma_{\sigma}\right) \\
& \quad+q_{\sigma}\left(\nabla^{2} \phi-\frac{\mu_{0} I \chi}{(2 \pi r)^{2}}+\mu_{0} \mathbf{J} \cdot \mathbf{u}_{\sigma}+\nabla \cdot(\eta \mathbf{J})\right)
\end{aligned}
$$

$$
+\nabla^{2} \Theta_{\sigma}=0
$$

Alternatively, the irrotational part of the motion can be described using Eq. (4) to obtain

$$
\begin{aligned}
\int d \mathbf{s} \cdot \nabla & {\left[m_{\sigma}\left(-\frac{\partial \Omega_{\sigma}}{\partial t}+\frac{u_{\sigma}^{2}}{2}-\frac{4}{3} v_{\sigma} \nabla \cdot \mathbf{u}_{\sigma}\right)+q_{\sigma} \phi+\Theta_{\sigma}\right] } \\
= & -\oint d \mathbf{l} \cdot\left[m_{\sigma}\left(-\chi_{\sigma} \mathbf{u}_{\sigma}+v_{\sigma} \nabla \chi_{\sigma}\right)\right. \\
& \left.+q_{\sigma}\left(-\mu_{0} I \mathbf{u}_{\sigma}+\eta \nabla I\right)\right]
\end{aligned}
$$

which gives the time evolution of $\Omega_{\sigma}$. The continuity equation gives the time evolution of the density,

$$
\frac{\partial n_{\sigma}}{\partial t}+u_{\sigma} \cdot \nabla n_{\sigma}+n_{\sigma} \Gamma_{\sigma}=0
$$

In contrast to the results obtained on taking the curl of Eq. (23), Eq. (35) does not lend itself to simple interpretation. It is possible, though, to observe that this equation contains sound waves [first and last terms in Eq. (35) balance, first and last terms in Eq. (36) balance], and also that it contains Ohm's law (coefficient of $q_{\sigma}$ forced to vanish). Because of the somewhat obscure nature of these irrotational equations, it is useful to consider a different point of view, namely that of magnetohydrodynamics where a different set of linear combinations of the equations of motion are used so that the dynamics are described by the center of mass velocity $\mathbf{U}$ and by the current density $\mathbf{J}$ instead of by the two-fluid velocities $\mathbf{u}_{i}$ and $\mathbf{u}_{e}$.

\section{COMPRESSIBLE MHD EQUATIONS WITH HALL TERM}

\section{A. Derivation of dimensionless MHD-like equations of motion}

In order to proceed to a set of MHD equations we drop electron inertia terms (i.e., let $m_{e} \rightarrow 0$ ) in the electron version of Eq. (23) so that the respective electron and ion equations of motion become

$$
\nabla\left(-e \phi+\Theta_{e}\right)-\frac{e}{2 \pi}\left[\nabla\left(\frac{\partial f}{\partial \iota}\right)-\mu_{0} I \mathbf{u}_{e}+\eta \nabla I\right] \times \nabla \theta=0
$$

and

$$
\begin{aligned}
& \nabla\left\{m_{i}\left(-\frac{\partial \Omega_{i}}{\partial t}+\frac{u_{i}^{2}}{2}-\frac{4}{3} v_{i} \nabla \cdot \mathbf{u}_{i}\right)+Z e \phi+\Theta_{i}\right\} \\
& +\frac{1}{2 \pi}\left\{\begin{array}{c}
m_{i}\left[\nabla\left(\frac{\partial \psi_{i}}{\partial t}\right)-\chi_{i} \mathbf{u}_{i}+v_{i} \nabla \chi_{i}\right] \\
+Z e\left[\nabla\left(\frac{\partial f}{\partial t}\right)-\mu_{0} I \mathbf{u}_{i}+\eta \nabla I\right]
\end{array}\right\} \times \nabla \theta=0 .
\end{aligned}
$$

The ion equation can be rearranged by adding $Z$ times the electron equation to it and using $\mathbf{u}_{i} \simeq \mathrm{U}, \Omega_{i} \simeq \Omega, \chi_{i}$ $\simeq \chi, \psi_{i} \simeq \psi, v_{i} \simeq \nu$ where $\mathbf{U}, \chi, \psi$, and $v$ are, respectively, the center of mass velocity, compressibility function, stream- 
function, vorticity function, and viscosity, i.e., $\nabla \times \mathbf{U}$ $=(2 \pi)^{-1} \chi \nabla \theta$. With these manipulations Eq. (38) becomes

$$
\begin{aligned}
& \nabla\left\{m_{i}\left(-\frac{\partial \Omega}{\partial t}+\frac{U^{2}}{2}-\frac{4}{3} \nu \nabla \cdot \mathbf{U}\right)+Z \Theta_{e}+\Theta_{i}\right\} \\
& \quad+\frac{1}{2 \pi}\left\{m_{i}\left[\nabla\left(\frac{\partial \psi}{\partial t}\right)-\chi \mathbf{U}+v \nabla \chi\right]-\frac{Z \mu_{0} I}{n_{e}} \mathbf{J}\right\} \times \nabla \theta=0 .
\end{aligned}
$$

The electron velocity in Eq. (37) can be eliminated using

$$
\mathbf{u}_{e} \simeq \mathbf{U}-\frac{1}{n_{e} e} \mathbf{J}
$$

to give

$$
\begin{aligned}
& \nabla\left(-e \phi+\Theta_{e}\right)-\frac{e}{2 \pi}\left[\nabla\left(\frac{\partial f}{\partial t}\right)-\mu_{0} I \mathbf{U}+\frac{\mu_{0} I}{n_{e} e} \mathbf{J}+\eta \nabla I\right] \times \nabla \theta \\
& =0 .
\end{aligned}
$$

Equations (39) and (41) are MHD-type equations since they involve only center of mass quantities and currents; no species velocities appear.

To proceed further it is useful to introduce dimensionless (tilde) variables defined in terms of the current $I_{c}$, magnetic field $B_{c}=\mu_{0} I_{d} / 2 \pi r_{c}$, mass density $\rho_{c}$, and Alfvén velocity $V_{\mathrm{Ac}}=B_{\mathrm{C}} \sqrt{\mu_{\mathrm{O}} \rho_{c}}$ at some characteristic location $r=r_{c}, z=z_{c}$. Thus, space and time variables become

$$
r-r_{c} \tilde{r}, \quad z=r_{c} \tilde{z}, \quad t=\frac{r_{c}}{V_{\mathrm{Ac}}} \widetilde{t}
$$

flux-like variables become

$$
\begin{aligned}
& \psi=V_{\mathrm{A} c} r_{c}^{2} \widetilde{\psi}, \quad \Omega=V_{\mathrm{A} c} r_{c} \widetilde{\Omega}, \quad \chi=V_{\mathrm{A} c} \chi, \\
& I=I_{c} \tilde{I}, \quad f=\mu_{0} I_{c} r_{c}^{2} \widetilde{f},
\end{aligned}
$$

vectors become

$$
\mathbf{U}=V_{\mathrm{Ac}} \widetilde{\mathbf{U}}, \quad \mathbf{B}=B_{c} \widetilde{I} \widetilde{\mathbf{V}} \theta, \quad \mathbf{A}=B_{c} r_{c} \widetilde{\mathbf{A}},
$$

and scalars related to irrotational dynamics become

$P=\frac{B_{c}^{2}}{\mu_{0}} \widetilde{P}, \quad \phi=V_{\mathrm{Ac}} B_{c} r_{c} \tilde{\phi}, \quad \rho=\rho_{c} \tilde{\rho}, \quad \Theta_{\sigma}=m_{i} V_{\mathrm{A} c}^{2} \widetilde{\Theta}_{\sigma}$.

Additionally we note that charge neutrality, $Z n_{i}=n_{e}$ gives

$$
\rho=\frac{m_{i} n_{e}}{Z}
$$

and also the ion plasma frequency becomes

$$
\omega_{p i}^{2}=\frac{n_{i} q_{i}^{2}}{m_{i} \epsilon_{0}}=\frac{Z n_{e} e^{2}}{m_{i} \epsilon_{0}} .
$$

Finally it is useful to define the Hall parameter (normalized ion collisionless skin depth)

$$
\alpha_{c}=\frac{c}{\omega_{p i c} r_{c}}
$$

where $\omega_{\text {pic }}$ means ion plasma frequency evaluated at $r=r_{c}, z=z_{c}$. Thus $\alpha_{c}$ is a constant and may be factored in and out of spatial derivatives as desired.

Substituting for all quantities in Eq. (39) using the above definitions, and dropping the tildes for clarity, the dimensionless ion equation of motion becomes

$$
\begin{aligned}
\nabla & \left.-\frac{\partial \Omega}{\partial t}+\frac{U^{2}}{2}+\frac{I^{2}}{2 \rho r^{2}}+Z \Theta_{e}+\Theta_{i}-\frac{4}{3 R} \nabla \cdot \mathrm{U}\right\} \\
& +\left[\frac{1}{2 \pi}\left[\nabla\left(\frac{\partial \psi}{\partial t}\right)-\chi \mathrm{U}+\frac{1}{R} \nabla \chi\right]\right. \\
& \left.+\frac{I^{2} r^{2}}{2} \nabla\left(\frac{1}{\rho r^{2}}\right) \times \nabla \theta\right\} \times \nabla \theta=0,
\end{aligned}
$$

where we have introduced the hydrodynamic Reynolds number

$$
R=\frac{V_{\mathrm{Ac}} r_{c}}{v}
$$

and also used the relation

$$
\begin{aligned}
& -\frac{I}{\rho}(\nabla I \times \nabla \theta) \times \nabla \theta \\
& \quad=\nabla\left(\frac{I^{2}}{2 \rho r^{2}}\right)+\frac{I^{2} r^{2}}{2}\left[\nabla\left(\frac{1}{\rho r^{2}}\right) \times \nabla \theta\right] \times \nabla \theta
\end{aligned}
$$

to separate out the scalar magnetic pressure term from the acceleration due to the total magnetic stress tensor $(\sim \mathbf{J} \times \mathbf{B} / \rho)$.

Similarly, substitution of the definitions of Eqs. (43)(48) into the electron equation, Eq. (41), gives the dimensionless electron equation of motion

$$
\begin{aligned}
& \nabla\left(-\phi+\alpha_{c} \frac{I^{2}}{2 \rho r^{2}}+Z \alpha_{c} \Theta_{e}\right) \\
& -\left\{\nabla \frac{\partial f}{\partial t}-I \mathrm{U}+\frac{1}{S} \nabla I\right. \\
& \left.-\alpha_{c} \frac{I^{2} r^{2}}{2}\left[\nabla\left(\frac{1}{\rho r^{2}}\right) \times \nabla \theta\right]\right\} \times \nabla \theta=0
\end{aligned}
$$

where we have introduced the magnetic Reynolds number

$$
S=\frac{\eta}{\mu_{0} V_{\mathrm{Ac}} r_{c}}
$$

\section{B. Rotational MHD equations}

Let us now consider the equations resulting from taking the curl and then dotting with $\nabla \theta$ on the dimensionless ion and electron equations of motion, Eqs. (49) and (51). Using Eq. (2) on Eq. (49) gives a vorticity transport equation

$$
\begin{aligned}
& \rho\left[\frac{\partial}{\partial t}\left(\frac{\chi}{2 \pi \rho r^{2}}\right)+\mathbf{U} \cdot \nabla\left(\frac{\chi}{2 \pi \rho r^{2}}\right)\right] \\
& \quad=\nabla \cdot\left(\frac{1}{2 \pi r^{2} R} \nabla \chi\right)+\nabla \theta \cdot \nabla\left(\frac{I^{2}}{2}\right) \times \nabla\left(\frac{1}{\rho r^{2}}\right),
\end{aligned}
$$


where we have used the relation $\nabla \cdot \mathbf{U}=-\rho^{-1}(\partial \rho / \partial t$ $+\mathbf{U} \cdot \nabla \rho)$ to write

$$
\frac{\partial}{\partial t}\left(\frac{\chi}{r^{2}}\right)+\nabla \cdot\left(\frac{\chi}{r^{2}} \mathbf{U}\right)=\rho\left[\frac{\partial}{\partial t}\left(\frac{\chi}{\rho r^{2}}\right)+\mathbf{U} \cdot \nabla\left(\frac{\chi}{\rho r^{2}}\right)\right] .
$$

In the incompressible limit where $\rho=1$, Eq. (53) becomes the vorticity transport equation discussed in Ref. 21, and the quantity $r^{-4} \partial I^{2} / \partial z$ acts as a volumetric source of vorticity (recall the factor of $2 \pi$ difference between the definitions of $\chi$ used here and in Ref. 21).

Similarly, using Eq. (2), the electron equation becomes

$$
\begin{aligned}
& \rho\left[\frac{\partial}{\partial t}\left(\frac{I}{\rho r^{2}}\right)+\mathrm{U} \cdot \nabla\left(\frac{I}{\rho r^{2}}\right)\right] \\
& \quad=\nabla \cdot\left(\frac{1}{r^{2} S} \nabla I\right)-\alpha_{c} \nabla \theta \cdot \nabla\left(\frac{I^{2}}{2}\right) \times \nabla\left(\frac{1}{\rho r^{2}}\right)
\end{aligned}
$$

which in the limit of incompressibility and zero Hall parameter becomes the current transport equation of Ref. 21 . Comparison of Eqs. (53) and (55) shows that the second terms on the rhs of both equations are identical except for a factor $-\alpha_{c}$; thus the magnitude of $\alpha_{c}$ gives the relative importance of (1) nonlinear Hall effects which, as shown by Fruchtman, ${ }^{19}$ give a faster than classical diffusion of flux into the plasma, and (2) vorticity generation, which result in bulk plasma flow.

Note that Eq. (55) is mathematically equivalent to the Hall MHD Ohm's law used by Fruchtman [cf. Eq. (2) of Ref. 19] but has been expressed in terms of the center of mass velocity $\mathbf{U}$ (rather than the electron fluid velocity $\mathbf{u}_{e}$ ) and nondimensional variables have been used. This equation [which is equivalent to the zero-mass limit of the electron version of Eq. (27) here] shows that flux is perfectly frozen into the electron fluid if there is zero resistivity. Although Eq. (55) is mathematically equivalent to Fruchtman's Eq. (2), the format of Eq. (55) has the virtue of explicitly showing that flux is frozen into the center of mass frame only if there is zero resistivity (i.e., $S \rightarrow \infty$ ) and also $\alpha_{c}=0$. If there is zero resistivity but $\alpha_{c}>0$, then Eq. (55) shows that flux does not convect with the center of mass, and so is not frozen into the center of mass frame. If $\mathbf{U}=0$ is assumed (as was done in Ref. 19) then Eq. (55) becomes ${ }^{19}$ a Burgers' type equation giving a fast, nonlinear flux "diffusion" in the lab frame; the finite $\alpha_{c}$ term is the Hall term. For nonstationary center of mass (i.e., $\mathbf{U} \neq 0$ ), Eq. (55) becomes a Burgers' equation in the center of mass frame and the term containing $\alpha_{c}$ gives fast, nonlinear Hall "diffusion" in the center of mass frame.

If $\alpha_{c} \ll 1$ then Hall diffusion will be relatively unimportant compared to vorticity generation, and vice versa if $\alpha_{c} \gg 1$. If $\alpha_{c} \sim 1$ then both effects will be equally important. As an example, the plasma erosion opening switch (PEOS) operates in a parameter range where Hall diffusion has been shown ${ }^{19}$ to be important; since $\alpha_{c} \sim 0.2$ for typical PEOS operation, comparison of Eqs. (53) and (55) shows that vorticity generation should also be important in the PEOS.

\section{Irrotational MHD equations}

Let us now use Eq. (4) on the ion and electron equations of motion, Eqs. (39) and (41), to obtain the equations governing irrotational quantities. The ion irrotational equation becomes

$$
\begin{aligned}
\int d \mathbf{s} & \cdot \nabla\left\{-\frac{\partial \Omega}{\partial t}+\frac{U^{2}}{2}+\frac{I^{2}}{2 \rho r^{2}}+Z \Theta_{e}+\Theta_{i}-\frac{4}{3 R} \nabla \cdot \mathbf{U}\right\} \\
= & -\oint d \mathbf{l} \cdot\left\{\left(-\chi \mathbf{U}+\frac{1}{R} \nabla \chi\right)\right. \\
& \left.+\pi I^{2} r^{2} \nabla\left(\frac{1}{\rho r^{2}}\right) \times \nabla \theta\right\}
\end{aligned}
$$

which in the incompressible limit becomes the pressure equation of Ref. 21. Similarly, the electron irrotational equation becomes

$$
\begin{array}{r}
\left.\int d \mathbf{s} \cdot \nabla \mid-\phi+\alpha_{c} \frac{I^{2}}{2 \rho r^{2}}+Z \alpha_{c} \Theta_{e}\right\} \\
=2 \pi \oint d \mathbf{l} \cdot\left\{-I \mathrm{U}+\frac{1}{S} \nabla I\right. \\
\left.-\alpha_{c} \frac{I^{2} r^{2}}{2}\left[\nabla\left(\frac{1}{\rho r^{2}}\right) \times \nabla \theta\right]\right\}
\end{array}
$$

which in the limit of zero Hall parameter becomes the equation for electrostatic potential of Ref. 21 .

Finally, the continuity equation can be written as

$$
\frac{\partial \rho}{\partial t}+\mathbf{U} \cdot \nabla \rho=\rho \nabla^{2} \Omega
$$

giving the time evolution of $\rho$.

These equations can be categorized as:

(1) equations that explicitly advance a scalar in time; there are four equations of this type, namely:

(a) Eq. (53) which advances the fluid circulation (vorticity) $\chi / 2 \pi \rho r^{2}$,

(b) Eq. (55) which advances the magnetic flux (current) $I / \rho r^{2}$,

(c) Eq. (56) which advances the compressibility scalar $\Omega$,

(d) Eq. (58) which advances the mass density $\rho$;

(2) equations which do not involve time derivatives, and which give other scalars in terms of the four scalars listed above:

(a) a Poisson-like equation for the streamfunction $\nabla \cdot\left(r^{-2} \psi\right)=-r^{-2} \chi$

(b) the specified dependence of pressure on density,

(c) Eq. (57) which determines the electrostatic potential.

This system of equations, together with temporal initial conditions and spatial boundary conditions, describes the time evolution of an arbitrary configuration having purely poloidal currents and axisymmetry. 


\section{DISCUSSION AND SUMMARY}

By separately considering the rotational and irrotational parts of the compressible two-fluid equations of motion of an axisymmetric plasma having purely poloidal currents we have shown that the Kelvin circulation theorem of classical fluid mechanics becomes generalized to being a conservation of the canonical circulation $S_{\sigma}=\oint\left(m_{\sigma} \mathbf{u}_{\sigma}\right.$ $\left.+q_{\sigma} \mathbf{A}\right) \cdot d \mathbf{l}$. By combining the two fluid equations to form MHD equations, it is further shown that there is a near symmetry between the source term for fluid vorticity in the vorticity transport equation and the nonlinear finite Hall term in the induction equation.

Note added in proof. A. A. Chernov and V. V. Yankov [Sov. J. Plasma Phys. 8, 522 (1982)], have also discussed the "frozen-in" condition $B / n r=$ const of an electron fluid element.

\section{ACKNOWLEDGMENT}

Supported by National Science Foundation Grant No. ECS-8814184.

${ }^{1} D$. Mosher and D. Colombant, Phys. Rev. Lett. 68, 2600 (1992).

${ }^{2}$ J. D. Sethian, A. E. Robson, K. A. Gerber, and A. W. de Silva, Phys. Rev. Lett. 59, 892 (1987).

${ }^{3}$ J. P. Chittenden and M. G. Haines, Phys. Fluids B 2, 1889 (1990).

${ }^{4}$ J. Marshall, Phys. Fluids 3, 135 (1960).

${ }^{5} \mathrm{~J}$. W. Mather, in Methods of Experimental Physics, Plasma Physics, edited by R. H. Lovberg and H. R. Griem (Academic, New York, 1971), Vol. 9, Part B, Chap. 15.

${ }^{6}$ E. J. T. Burns, S. M. Falacy, R. A. Hill, P. D. Thacher, H. A. Koehler, B. Davis, and R. E. Shafer, Phys. Lett. 133, 144 (1988).

${ }^{7}$ H. Maecker, Z. Phys. 141, 198 (1955).

${ }^{8} \mathrm{G}$. R. Jones, High Pressure Arcs in Industrial Devices (Cambridge University Press, Cambridge, 1 1988).

${ }^{9} J$. W. McKelliget and J. Szekely, J. Phys. D. 16, 1007 (1983).

${ }^{10}$ K. R. Stalder and R. L. Sharpless, J. Appl. Phys. 68, 6187 (1990).

${ }^{11}$ R. M. Myers, M. A. Mantenieks, and M. R. LaPointe, in Proceedings of the AIAA/NASA/OAI Conference on Advanced SEI Technologies. 1991, Cleveland, Ohio (American Institute of Aeronautics and Astronautics, New York, 1991), Paper No. AIAA 91-3568.

${ }^{12}$ D. J. Heimerdinger and M. Martinez-Sanchez, J. Prop. 7, 975 (1991).

${ }^{13}$ G. Kirkman-Amemiya and M. A. Gundersen, Appl. Phys. Lett. 60, 316 (1992).

${ }^{14}$ G. Cooperstein and P, F. Otținger, IEEE Trans. Plasma Sci. PS-15, 629 (1987).

${ }^{15}$ D. Hinshelwood, B. Weber, J. M. Grossman, and R. J. Commisso, Phys. Rev. Lett. 68, 3567 (1992).

${ }^{16}$ R. D. Blandford, in Quasars: IAU Symposium 119, edited by G. Swarup and V. K. Kaphahi (Reidel, Dordrecht, Netherlands, 1986), p. 359, and especially p. 369.

${ }^{17}$ G. Benford, in Astrophysical Jets, edited by A. Ferrari and A. G. Pacholczyk (Reidel, Dordrecht, Netherlands, 1983), pp. 271 and 279.

${ }^{18}$ R. M. Kulsrud, P. F. Ottinger, and J. M. Grossman, Phys. Fluids 31, 1741 (1988).

${ }^{19}$ A. Fruchtman, Phys. Fluids B 4, 3446 (1992).

${ }^{20}$ A. S. Kingsep, Yu. V. Mohkov, and K. V. Chukbar, Sov. J. Plasma Phys. 10, 495 (1984).

${ }^{21}$ P. M. Bellan, Phys. Rev. Lett. 69, 3515 (1992).

${ }^{22}$ A. B. Hassam and J. D. Huba, Phys. Fluids 31, 320 (1988). 